\title{
ATIC/PAMELA anomaly from fermionic decaying Dark Matter
}

\author{
Chuan-Hung Chen ${ }^{1,2 *}$, Chao-Qiang Geng ${ }^{3 \dagger}$ and Dmitry V. Zhuridov ${ }^{3 \ddagger}$ \\ ${ }^{1}$ Department of Physics, National Cheng-Kung University, Tainan 701, Taiwan \\ ${ }^{2}$ National Center for Theoretical Sciences, Hsinchu 300, Taiwan \\ ${ }^{3}$ Department of Physics, National Tsing-Hua University, Hsinchu 300, Taiwan
}

(Dated: July 27, 2021)

\begin{abstract}
We demonstrate that an economical two Higgs doublet model can explain the electron and positron excesses in the recent ATIC and PAMELA experiments by the three body decays of the dark matter (DM) fermions without requiring the fine turning of the couplings and degeneracy of masses. We also show that the mass and lifetime of the decaying DM particle may not be fixed to be around $1 \mathrm{TeV}$ and $10^{26} \mathrm{sec}$, respectively. Moreover, we note that this model includes a stable dark matter candidate as well.
\end{abstract}

\footnotetext{
* Email: physchen@mail.ncku.edu.tw

† Email: geng@phys.nthu.edu.tw

‡ Email: zhuridov@phys.nthu.edu.tw
} 
The observed neutrino oscillations [1] and evidence for dark matter (DM) [1] imply physics beyond the standard model. In addition, recently reported PAMELA [2]/ATIC [3] cosmicray measurements show the positron/electron excess above the calculated background for the energy of order $100 \mathrm{GeV}$. These data is consistent with the previous measurements of the high energy electrons and positrons fluxes in the cosmic ray spectrum by PPB-BETS [4], HEAT [5] and AMS [6]. The, so-called, PAMELA/ATIC (P/A) anomaly can be explained by either annihilations or rare decays of DM particles. However, the possibility of the DM annihilations requires a boost factor of order $10^{2}-10^{3}$ to make it consistent with the thermally averaged annihilation cross section obtained from the observed relic density, whereas the analysis of the DM distribution indicates that the most probable boost factor should be of order 10 [7, 8]. On the other hand, the long enough lifetime of the decaying DM is achieved by using either some arbitrary small couplings (see Refs. [8, 9, 10, 11] and references therein) or large scale suppressions associated with high-dimensional operators at the low energy in the contexts of supersymmetry [12], technicolor [13], hidden gauge boson [14] and hidden fermion [15] models. In particular, DM decays through three-body channels have been discussed in Refs. [12, 15]. In this letter, we would like to explain the $\mathrm{P} / \mathrm{A}$ result by three-body decays of DM fermions in a simple extended two-Higgs doublet model.

We introduce two neutral leptons $N_{k}$ with the masses $M_{k}(k=1,2)$ and second doublet scalar $\eta$ with the mass $M_{\eta}$ in addition to the SM particles. We assume that new particles are odd under a $Z_{2}$ symmetry. Note that the same particle content can be used to explain the small neutrino masses with either stable DM [16] or leptogenesis [17]. The new Majorana mass term and Yukawa couplings can be written as

$$
M_{k} N_{k} N_{k}+y_{i k} \bar{L}_{i} \eta N_{k}+\text { H.c. }
$$

where $L$ is the lepton doublet and $i$ and $k$ are the flavor indexes. We consider the masses of $M_{1}<M_{2}<M_{\eta}$. Hence, $N_{1}$ is stable and $N_{2}$ can only decay to three, as shown in Fig. 1, or more particles.

The lifetime of $N_{2}$ is given by

$$
\tau_{N_{2}} \simeq \frac{1}{\Gamma\left(N_{2} \rightarrow N_{1} \ell_{i}^{ \pm} \ell_{j}^{\mp}\right)}=\frac{128(2 \pi)^{3}}{3} \frac{M^{4} M_{2}^{3}}{\left(M_{21}^{2}\right)^{4}},
$$

where $M_{21}^{2}=M_{2}^{2}-M_{1}^{2}$ is the DM leptons mass splitting and $M \equiv M_{\eta} / y$ with $y \equiv\left|y_{i k}\right|$. The energy distribution of electrons/positrons produced in a single three body decay of $N_{2}$ 


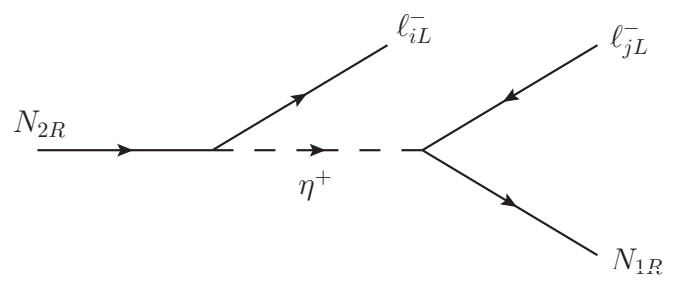

FIG. 1: Diagram for the DM decay.

can be written as

$$
\frac{d N_{e}}{d E}=\frac{72 M_{2}^{3}}{\left(M_{21}^{2}\right)^{4}}\left(M_{21}^{2}-\frac{16}{9} M_{2} E\right) E^{2} .
$$

We use the same method to calculate the electron/positron flux as that in Refs. [18, 19, 20]. The DM component of the primary electron/positron flux is given by

$$
\Phi_{e}^{D M}(E)=\frac{c}{4 \pi M_{2} \tau_{N_{2}}} \int_{0}^{M_{21}^{2} /\left(2 M_{2}\right)} d E^{\prime} G\left(E, E^{\prime}\right) \frac{d N_{e}}{d E^{\prime}}
$$

where $E$ is in units of $\mathrm{GeV}$ and $c$ is the speed of light. All the information about astrophysics is encoded in the Green function $G\left(E, E^{\prime}\right)$, approximately given by

$$
G\left(E, E^{\prime}\right) \simeq \frac{10^{16}}{E^{2}} \exp \left[a+b\left(E^{\delta-1}-E^{\prime \delta-1}\right)\right] \theta\left(E^{\prime}-E\right) \quad\left[\mathrm{cm}^{-3} \mathrm{~s}\right]
$$

The coefficients of $a$ and $b$ [9] for the spherically symmetric Navarro, Frenk and White (NFW) density profile of the DM in our Galaxy [21] and the diffusion parameter $\delta$ are listed in Table I for the three propagation models of M1, MED and M2 [22], respectively, which are consistent with the observed Boron-to-Carbon ratio [23]. Here, we have neglected the charge-sign dependent solar modulation [24] and other astrophysical uncertainties [22], which could be significant at the energies below $10 \mathrm{GeV}$.

TABLE I: Coefficients of the approximate positron Green function of the NFW halo profile and the diffusion parameter $\delta$ for the propagation models of M1, MED and M2, respectively.

\begin{tabular}{|c|c|c|c|}
\hline Model & $\delta$ & $a$ & $b$ \\
\hline M1 & 0.46 & -0.9809 & -1.1456 \\
MED & 0.70 & -1.0203 & -1.4493 \\
M2 & 0.55 & -0.9716 & -10.012 \\
\hline
\end{tabular}


The total electron and positron fluxes are

$$
\Phi_{e^{-}}=\xi \Phi_{e^{-}}^{p r i m}+\Phi_{e^{-}}^{D M}+\Phi_{e^{-}}^{s e c}
$$

and

$$
\Phi_{e^{+}}=\Phi_{e^{+}}^{D M}+\Phi_{e^{+}}^{s e c}
$$

respectively, where $\Phi_{e^{-}}^{\text {prim }}$ is a primary astrophysical component, presumably originated from supernova remnants, $\Phi_{e^{-(+)}}^{D M}$ is an exotic primary component from DM decays, $\Phi_{e^{-(+)}}^{s e c}$ is a secondary component from the spallation of cosmic rays on the interstellar medium, and $\xi$ is a free parameter about 1 to fit the data when no DM primary source exists. We take $\xi=0.7$ to insure the flux calculation to be consistent with the ATIC data. For the background fluxes, we will use the parametrizations obtained in Refs. [24, 25], given by

$$
\begin{aligned}
\Phi_{e^{-}}^{\text {prim }}(E) & =\frac{0.16 E^{-1.1}}{1+11 E^{0.9}+3.2 E^{2.15}} \quad\left[\mathrm{GeV}^{-1} \mathrm{~cm}^{-2} \mathrm{~s}^{-1} \mathrm{sr}^{-1}\right] \\
\Phi_{e^{-}}^{\text {sec }}(E) & =\frac{0.7 E^{0.7}}{1+110 E^{1.5}+600 E^{2.9}+580 E^{4.2}} \quad\left[\mathrm{GeV}^{-1} \mathrm{~cm}^{-2} \mathrm{~s}^{-1} \mathrm{sr}^{-1}\right], \\
\Phi_{e^{+}}^{\text {sec }}(E) & =\frac{4.5 E^{0.7}}{1+650 E^{2.3}+1500 E^{4.2}} \quad\left[\mathrm{GeV}^{-1} \mathrm{~cm}^{-2} \mathrm{~s}^{-1} \mathrm{sr}^{-1}\right],
\end{aligned}
$$

where $E$ is in units of GeV. In Fig. 2, we show the electron energy spectra (left) and the
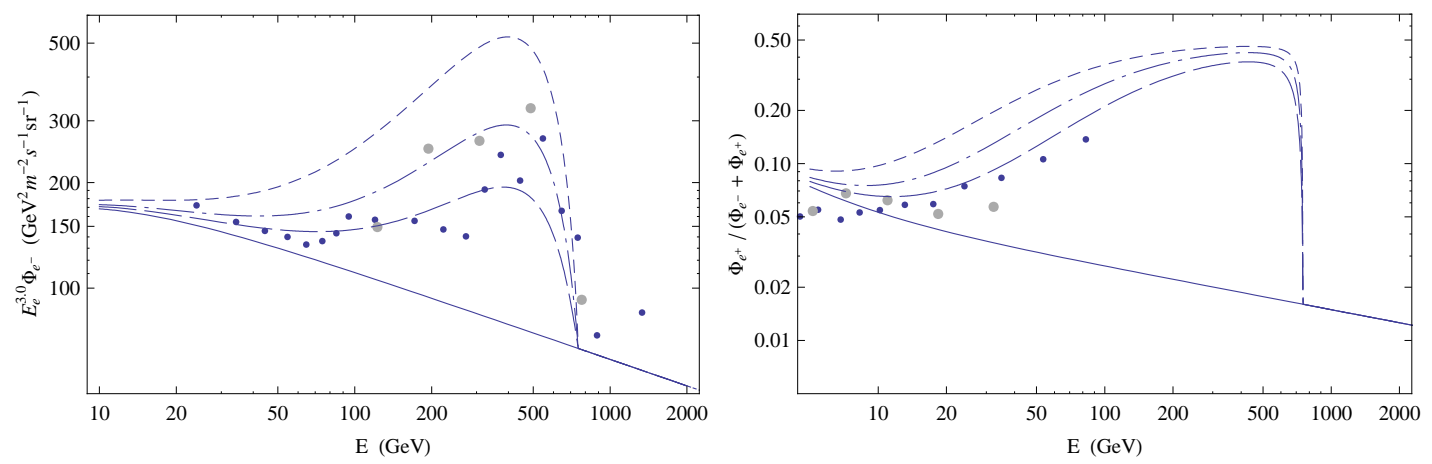

FIG. 2: Electron energy spectra (left) and positron fractions (right) of the DM decays, where $M_{1}=10 \mathrm{GeV}, M_{2}=1.5 \mathrm{TeV}, \tau_{2} \sim 10^{26} \mathrm{~s}$, short-dashed, dot-dashed and long-dashed lines represent $M=2.5 \times 10^{15}, 3 \times 10^{15}$ and $3.5 \times 10^{15} \mathrm{GeV}$, small-black and large-gray dots stand for the observations of ATIC and PPB-BETS (left) and PAMELA and HEAT (right), and solid lines correspond to the backgrounds calculated from Eqs. (8)-(10), respectively. 

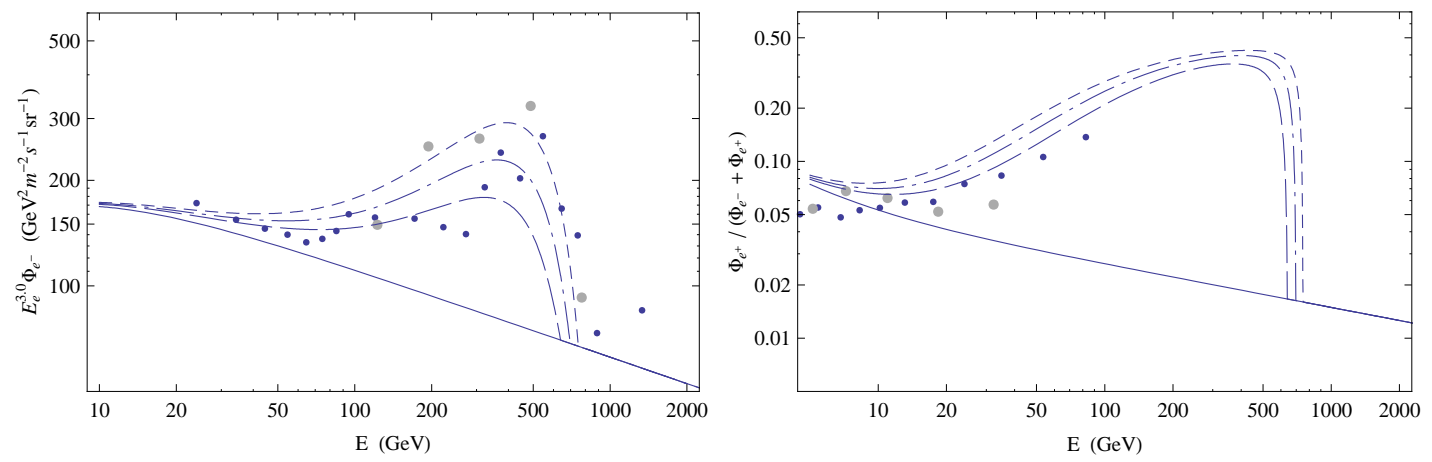

FIG. 3: Legend is the same as Fig. 2 but $M=3 \times 10^{15} \mathrm{GeV}, M_{2}=2 \mathrm{TeV}$ and short-dashed, dot-dashed and long-dashed lines represent $M_{1}=1,1.1 \mathrm{TeV}$ and $1.2 \mathrm{TeV}$, respectively.
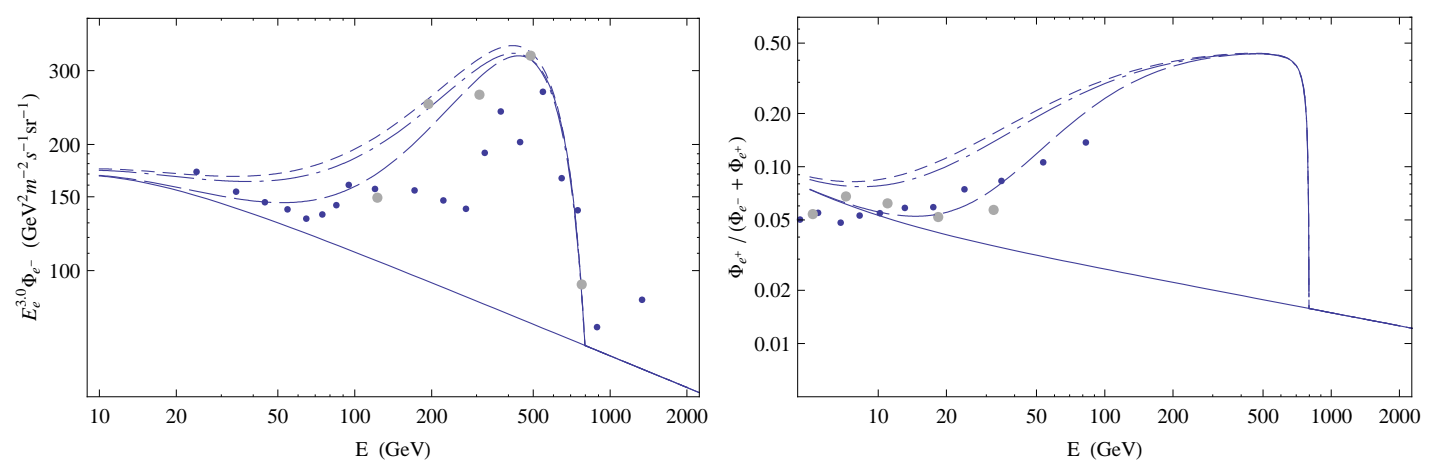

FIG. 4: Electron energy spectra (left) and positron fractions (right) for the decays of DM particles with $M_{2}=100 \mathrm{TeV}, \tau_{2}=10^{24} \mathrm{~s}$ and $M_{1}=(100-0.8) \mathrm{TeV}$ (dashed lines) where small-black and large-gray dots stand for the observations of ATIC and PPB-BETS (left) and PAMELA and HEAT (right), and solid, short-dashed, dot-dashed and long-dashed lines correspond to the backgrounds, M1, MED and M2, respectively.

positron fractions (right) of $N_{2} \rightarrow N_{1} \ell_{i}^{ \pm} \ell_{j}^{\mp}$ decays for $M_{1}=10 \mathrm{GeV}, M_{2}=1.5 \mathrm{TeV}$ and $M=2.5 \times 10^{15} \mathrm{GeV}$ (short-dashed line), $3 \times 10^{15} \mathrm{GeV}$ (dot-dashed line) and $3.5 \times 10^{15} \mathrm{GeV}$ (long-dashed line), while Fig. 3 for $M=3 \times 10^{15} \mathrm{GeV}, M_{2}=2 \mathrm{TeV}$ and $M_{1}=1 \mathrm{TeV}$ (short-dashed line), 1.1 TeV (dot-dashed line) and 1.2 TeV (long-dashed line), respectively. Here, the MED propagation model has been used and the background is represented by solid lines. In the above cases, the lifetime of $N_{2}$ is of order $10^{26} \mathrm{~s}$, while the values of $M$ can be achieved by taking $y_{i k} \sim 10^{-3}$ and $M_{\eta} \sim 10^{12} \mathrm{GeV}$. As seen from Figs. 2] and 3, the electron energy spectrum is more sensitive to the parameters in the model in contrast the positron 
fraction. The $\mathrm{P} / \mathrm{A}$ anomaly can be explained by the decays of $N_{2}$ with the mass higher than 1.5 TeV. We remark that for a lighter DM particle, the drop in the electron flux occurs at a lower energy compared to the ATIC data. In this sense, our mechanism is complementary to the annihilations of the DM fermions with masses below $1 \mathrm{TeV}$, shown in Refs. [26, 27]. Our model has more freedom in DM masses and lifetimes than the models with dominated twobody decays of DM particles since the drop in the electron flux in our case is determined by $M_{21}^{2} /\left(2 M_{2}\right)$ rather than (DM mass)/2, see Refs. [8, 9, 10, 11] and references therein. Hence, the DM lifetime should be only higher than the age of the universe $\left(4.3 \times 10^{17} \mathrm{~s}\right)$. However, for higher $M_{i}$, a mass degeneracy is needed. For example, to have $\tau_{2}=10^{24} \mathrm{~s}$, we need $M_{2}=10^{5} \mathrm{GeV}$ and $M_{1}=\left(10^{5}-800\right) \mathrm{GeV}$. The corresponding electron energy spectra and positron fractions are shown in Fig. 4 for the three propagation models of M1, MED and M2, respectively. We note that at the energies higher $400 \mathrm{GeV}$ the signals are not sensitive to the propagation models. Production mechanisms of the DM leptons in the early universe and the upper bounds on their masses from the $\gamma$-rays observations will be considered elsewhere [29].

Finally, we remark that future collider bounds on the masses of heavy neutrinos [28] are not applicable in this Letter due to the $Z_{2}$ symmetry. However, our model can be verified by precise measurements of the electron spectrum and positron fraction, since the shapes of the corresponding curves are not very flexible. In particular, future measurements of the positron fraction at energies higher than $100 \mathrm{GeV}$ can be crucial in testing the same origin of the ATIC and PAMELA electron and positron excesses.

In conclusion, we have investigated a new mechanism to generate the positron/electron excess from the decays of DM leptons. We have shown that the observed P/A anomaly can be explained by the three body decay of the neutral lepton $N_{2}$ with the mass $M_{2} \gtrsim 1.5 \mathrm{TeV}$ and the lifetime $10^{17} \mathrm{~s} \ll \tau_{2} \lesssim 10^{26}$ s. One of the advantages of our mechanism is that there are no requirements for the degeneracy of masses and unnaturally small couplings or any other enhancement factors.

\section{Acknowledgements}

This work is supported in part by the National Science Council of R.O.C. under Grant Nos: NSC- 97-2112-M-006-001-MY3 and NSC-95-2112-M-007-059-MY3. One of us (DVZ) 
would like to thank Prof. Kaoru Hagiwara for the hospitality at KEK and the exchange program between HEP at Taiwan and KEK.

[1] C. Amsler et al. [PDG Collaboration], Phys. Lett. B 667 (2008) 1.

[2] O. Adriani et al. [PAMELA Collaboration], Nature 458 (2009) 607.

[3] J. Chang et al. [ATIC Collaboration], Nature 456 (2008) 362.

[4] S. Torii et al. [PPB-BETS Collaboration], arXiv:0809.0760 [astro-ph].

[5] S. W. Barwick et al. [HEAT Collaboration], Astrophys. J 482 (1997) L191.

[6] M. Aguilar et al. [AMS-01 Collaboration], Phys. Lett. B 646 (2007) 145.

[7] J. Lavalle, Q. Yuan, D. Maurin and X. J. Bi, Astron. Astrophys. 479 (2008) 427.

[8] W. L. Guo and Y. L. Wu, arXiv:0901.1450 [hep-ph].

[9] A. Ibarra and D. Tran, arXiv:0811.1555 [hep-ph].

[10] I. Gogoladze, R. Khalid, Q. Shafi and H. Yüksel, arXiv:0901.0923 [hep-ph].

[11] K. Hamaguchi, F. Takahashi and T. T. Yanagida, arXiv:0901.2168 [hep-ph]; C. R. Chen, F. Takahashi and T. T. Yanagida, arXiv:0811.0477 [hep-ph].

[12] A. Arvanitaki et al., arXiv:0812.2075 [hep-ph].

[13] E. Nardi, F. Sannino, A. Strumia, arXiv:0811.4153 [hep-ph].

[14] C. R. Chen, M. M. Nojiri, F. Takahashi and T. T. Yanagida, arXiv:0811.3357 [astro-ph].

[15] K. Hamaguchi, S. Shirai and T. Yanagida, arXiv:0812.2374 [hep-ph].

[16] E. Ma, Phys. Rev. D 73 (2006) 077301.

[17] P. Gu and U. Sarcar, arXiv:0811.0956 [hep-ph].

[18] A. Ibarra and D. Tran, JCAP 0807 (2008) 002.

[19] C. R. Chen, F. Takahashi and T. Yanagida, Phys. Lett. B 671 (2009) 71.

[20] C. R. Chen and F. Takahashi, JCAP 0902 (2009) 004.

[21] J. F. Navarro, C. S. Frenk and S. D. M. White, Astrophysics J. 462 (1996) 563.

[22] T. Delahaye, R. Lineros, F. Donato, N. Fornengo and P. Salati, Phys. Rev. D 77 (2008) 063527.

[23] D. Maurin, F. Donato, R. Taillet and P. Salati, Astrophys. J. 555 (2001) 585.

[24] E. Blatz and J. Edsjo, Phys. Rev. D 59 (1999) 023511.

[25] I. Moskalenko and A. Strong, Astrophys. J. 493 (1998) 694. 
[26] X. J. Bi, P. H. Gu, T. Li, X. Zhang, arXiv:0901.0176[hep-ph].

[27] Q. H. Cao, E. Ma and G. Shaughnessy, arXiv:0901.1334 [hep-ph].

[28] C. S. Chen, C. Q. Geng and D. V. Zhuridov, Phys. Lett. B 666 (2008) 340; A. Ali, A. V. Borisov and D. V. Zhuridov, Phys. Atom. Nucl. 68 (2005) 2061 [Yad. Fiz. 68 (2005) 2123]; In: Particle Physics in Laboratory, Space and Universe (Singapore, World Scientific, 2005) P. 66 hep-ph/0512005; A. Ali, A. V. Borisov and N. B. Zamorin, Eur. Phys. J. C 21 (2001) 123.

[29] C. H. Chen, C. Q. Geng and D. V. Zhuridov, in progress. 